\title{
Centimeter-scale surface interactions using hydrodynamic flow confinements
}

\author{
David P. Taylor, Ismael Zeaf, Robert D. Lovchik, and Govind V. Kaigala* \\ IBM Research - Zürich, Säumerstrasse 4, CH-8803 Rüschlikon, Switzerland \\ Supplementary information
}

\author{
Corresponding author: \\ Dr. Govind Kaigala \\ IBM Research GmbH \\ Säumerstrasse 4 \\ CH-8803 Rüschlikon, Switzerland \\ Phone: +41 447248289 \\ E-mail: gov@zurich.ibm.com
}




\section{S1. Localized IgG assay on PDMS}

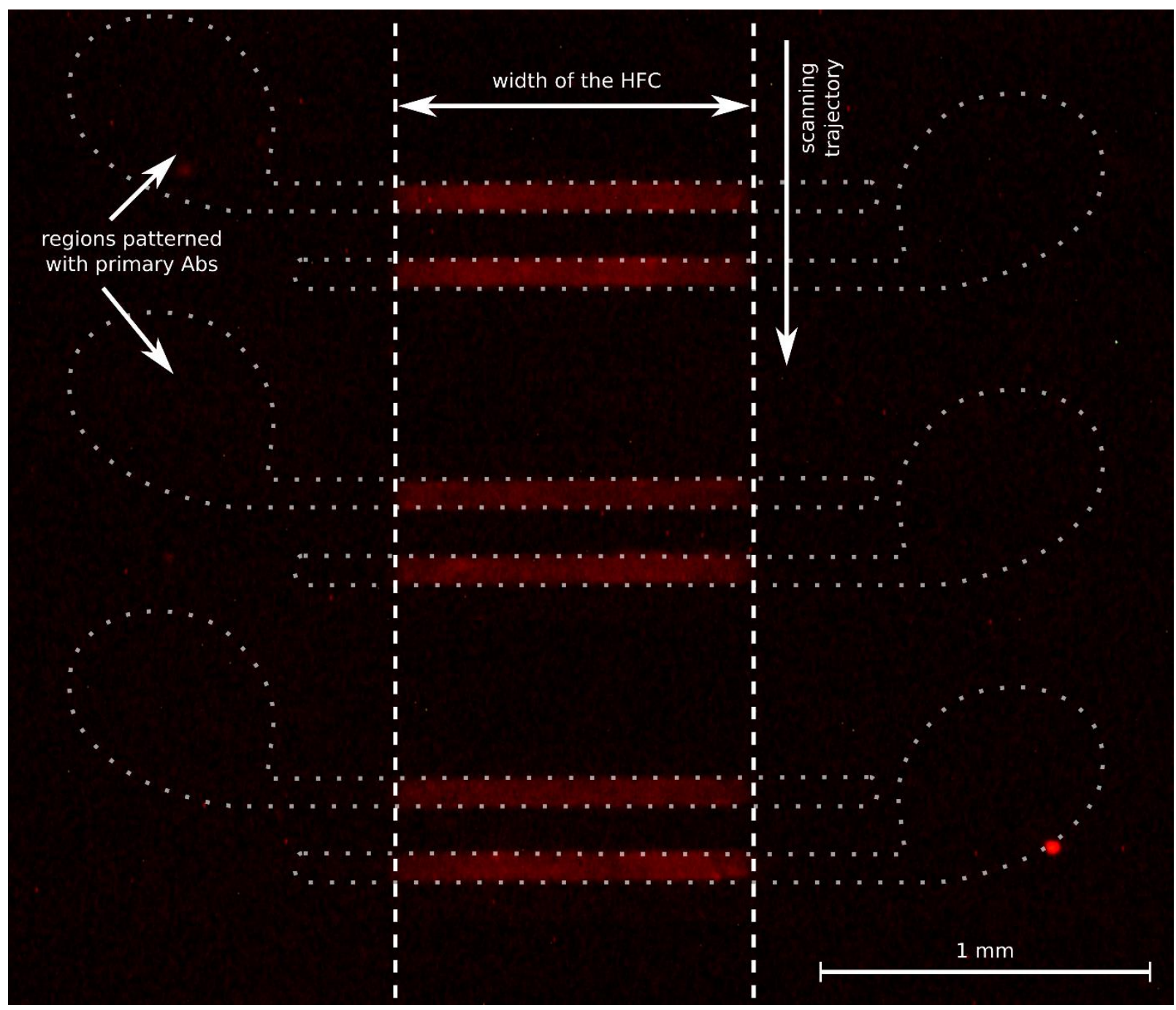

Figure S1. Localized pattering of a substrate using mm-scale HFC. A microfluidic stencil [30] was used to create a line-pattern of IgG from rabbit serum on a PDMS surface. A probe with slitaperture design creating an HFC with a width of $1.2 \mathrm{~mm}$ was scanned perpendicularly to the line pattern $\left(Q_{I}=6 \mu \mathrm{l} / \mathrm{min}, Q_{I} / Q_{A}=1 / 5\right.$, speed $0.02 \mathrm{~mm} / \mathrm{s}$, gap $\left.40 \mu \mathrm{m}\right)$ to expose only the center of the pattern to the processing liquid. The processing liquid in the HFC was PBS containing $\alpha$ Rabbit IgG labelled with rhodamine B at a concentration of $150 \mu \mathrm{g} / \mathrm{ml}$ and $1 \%$ of BSA. 


\section{S2. Setup for localized processing of immersed surfaces}

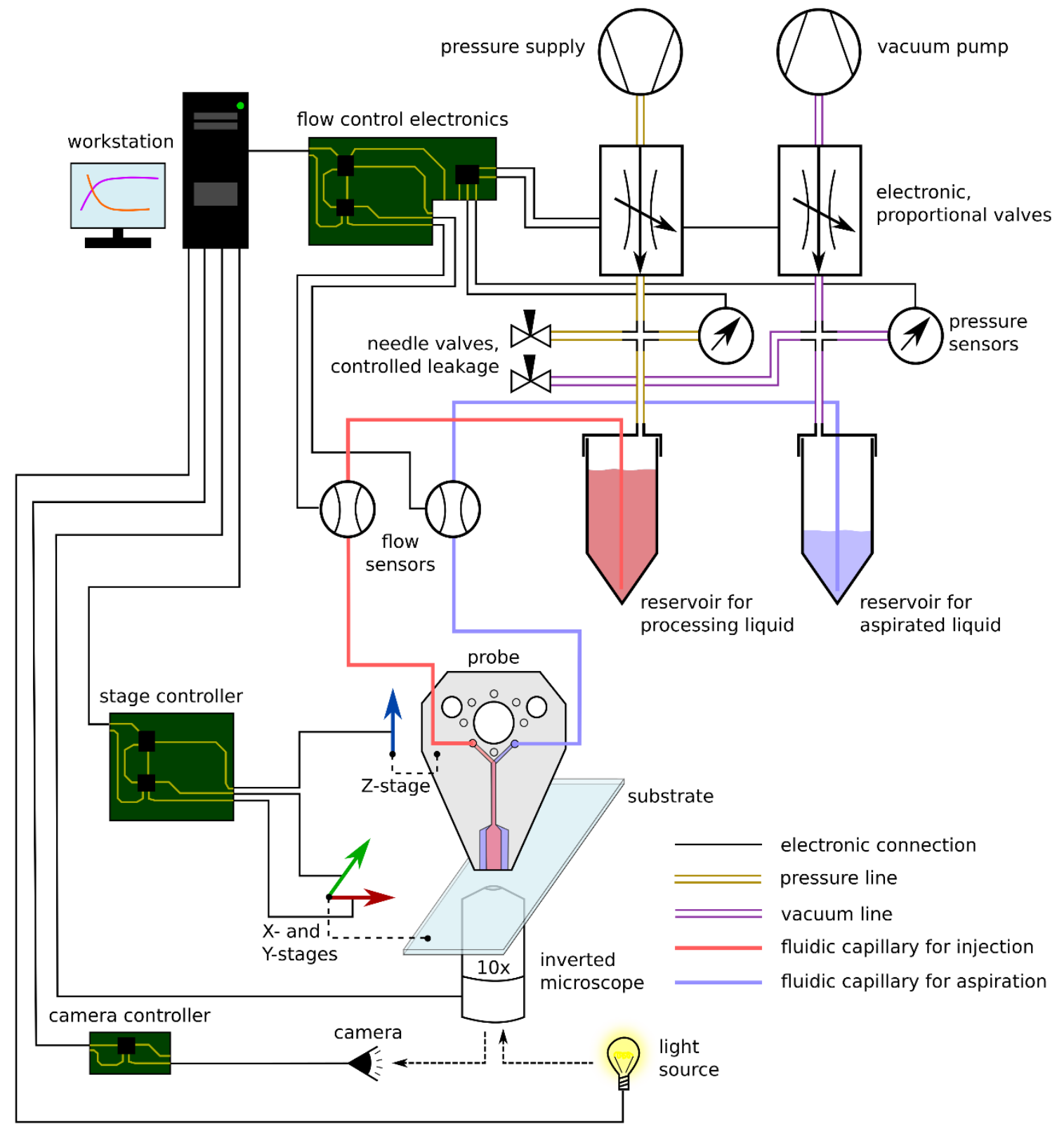

Figure S2. Schematic illustration of the setup. For activation of flows, the pressure or vacuum can be adjusted in the reservoirs connected to the probe. A stable adjustment of the pressure is possible, by regulating gas flow from a source with a proportional valve in combination with a controlled leakage to ambient to compensate for overshooting. Liquid flow is monitored by high precision flow sensors and the flow rates are used as feedback to adjust the pressure values in closed loop control. The substrate is supported by a stage that allows scanning in X-and Ydirection, while the probe is aligned with the optical axis of the microscope and can be moved in Z-direction for adjusting the gap between the probe and the substrate. 
S3. Reagent loading platform for multiplexing using aperture array designs

a)
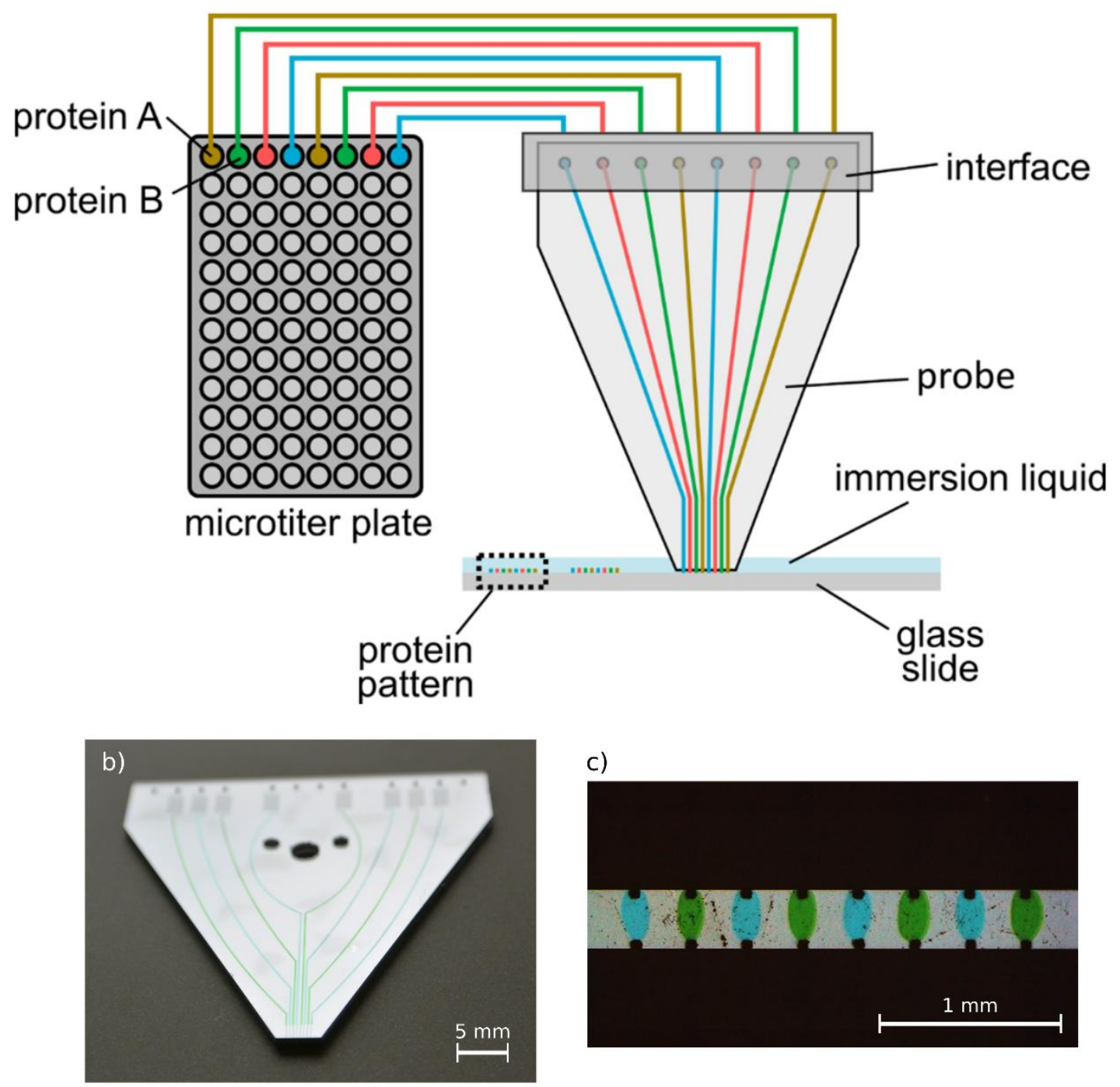

c)

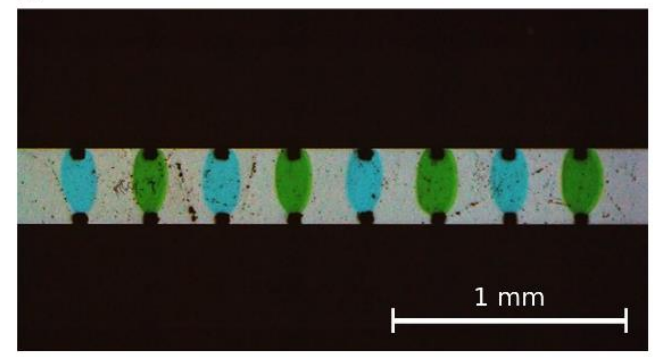

Figure S3. Sample loading and interfacing for probes with aperture-array designs. a) To allow for confinement of up to eight different processing liquids in parallel, the probe must be connected to a corresponding number of reservoirs individually. To enable simple loading and handling of processing liquids, we interfaced the aperture-array probe with 96-well microtiter plates: after loading of the processing liquids, the 96 well plate is inserted into a pressure chamber. The lid of the chamber holds eight capillaries, which upon closing is inserted into the wells containing the processing liquids. When the chamber is pressurized, the processing liquids are injected into the capillaries, which are interfaced with the probe via a 12 port linear connector (Dolomite Microfluidics Ltd., UK). b) Photograph of an aperture-array probe. Hydrodynamic resistors, for enabling operation in the ideal working range of the pressure control system, as well as fluidic connection ports are visible at the top end of the probe. c) Bright-field image of eight individual flows of processing liquids confined at the apex of an aperture-array probe. The processing liquids contain blue and green food dye for visualization. 